Article

\title{
Preparation and Properties of Self-Healing Waterborne Polyurethane Based on Dynamic Disulfide Bond
}

\author{
Gongbo Ye and Tao Jiang *
}

check for

updates

Citation: Ye, G.; Jiang, T. Preparation and Properties of Self-Healing

Waterborne Polyurethane Based on Dynamic Disulfide Bond. Polymers 2021, 13, 2936. https://doi.org/ $10.3390 /$ polym 13172936

Academic Editors: Wei Wu, Hao-Yang Mi, Chongxing Huang, Hui Zhao and Tao Liu

Received: 11 August 2021

Accepted: 26 August 2021

Published: 31 August 2021

Publisher's Note: MDPI stays neutral with regard to jurisdictional claims in published maps and institutional affiliations.

Copyright: (c) 2021 by the authors. Licensee MDPI, Basel, Switzerland. This article is an open access article distributed under the terms and conditions of the Creative Commons Attribution (CC BY) license (https:/ / creativecommons.org/licenses/by/ $4.0 /)$.
Hubei Collaborative Innovation Center for Advanced Organic Chemical Materials, Ministry of Education Key Laboratory for the Green Preparation and Application of Functional Materials, School of Materials Science and Engineering, Hubei University, Wuhan 430062,China; 2015011113000048@stu.hubu.edu.cn

* Correspondence: jiangtao@hubu.edu.cn

Abstract: A self-healing waterborne polyurethane (WPU) materials containing dynamic disulfide (SS) bond was prepared by introducing SS bond into polymer materials. The zeta potential revealed that all the synthesized WPU emulsions displayed excellent stability, and the particle size of them was about $100 \mathrm{~nm}$. The characteristic peaks of N-H and S-S in urethane were verified by FTIR, and the chemical environment of all elements were confirmed by the XPS test. Furthermore, the tensile strength, self-healing process and self-healing efficiency of the materials were quantitatively evaluated by tensile measurements. The results showed that the self-healing efficiency could reach $96.14 \%$ when the sample was heat treated at $70{ }^{\circ} \mathrm{C}$ for $4 \mathrm{~h}$. In addition, the material also showed a good reprocessing performance, and the tensile strength of the reprocessed film was $3.39 \mathrm{MPa}$.

Keywords: waterborne polyurethane; self-healing; dynamic disulfide bond

\section{Introduction}

To date, polymer materials have been widely used in various fields because of their excellent corrosion resistance and mechanical and barrier properties. However, it is accessible to produce micro-slaps on the surface and inside of the traditional polymer materials due to the influence of mechanical, light and chemical substances in the manufactural processing and practical applications. These cracks are difficult to detect with the naked eye and may cause further damage to the materials, thus reducing the mechanical properties and safety of the materials and shortening their service time. Therefore, some researchers introduced the biological self-healing characteristics and mechanisms into polymer materials and formed functional self-healing materials through bionic design. These materials can recover their original properties spontaneously after damage or under external stimulation, resulting in new intelligent/smart materials with longer lives and more reliable performances, such as shaped memory polymer [1-3] and self-healing polymer [4,5] intelligent polymer materials.

From the perspective of bionics, self-healing materials can be divided into extrinsic and intrinsic types according to whether they contain additional repair reagents or not. Extrinsic self-healing can preinstall some special structural components (such as microcapsules or micro-vessels) in the polymer composites by implantation technology. When the material is damaged, the repair reagent at the damaged part is released rapidly, which promotes the polymerization reaction and repairs the material structure in time [6], whereas intrinsic self-healing materials can achieve multiple internal repairs through the breaking and recombination of reversible chemical bonds within or between molecules. Therefore, it is not necessary to add additional repair reagents in advance $[7,8]$.

As the earliest discovered dynamic polymerization reaction, disulfide exchange reaction can be carried out at a low temperature and has great advantages in preparing dynamic polymers [9,10] For instance, aromatic disulfide compounds can form a rapid self-healing system [11]. In 2007, Nitschke et al. found that disulfide bonds in aromatic 
disulfides were easier to reach the translocation exchange equilibrium than that in aliphatic disulfides [12]. Ibon Odriozola et al. successfully prepared a catalyst-free disulfide bond polymer elastomer that can be repaired at room temperature by the condensation reaction of 4, 4'-dithiodianiline with isocyanate [13]. Kim et al. developed a transparent and easy-to-process polyurethane elastomer (IP-SS), which can be rapidly self-healed at room temperature and possess a maximum tensile strength of $6.8 \mathrm{MPa}$ [14]. Zhang et al. prepared a self-healing polyurethane material based on solar light-induced disulfide bond metathesis reaction [15]. In addition to the electron-donating groups connected with disulfide bonds, it helps to reduce their bond energy and form hydrogen bonds between macromolecular chains, the amorphous structures of aliphatic monomers and soft and hard segments also play a key role in the self-healing of hydrogen sulfide. However, there are still some problems in the development of the disulfide exchange reaction. Aromatic disulfide monomers are usually expensive and not conducive to large-scale industrial production. At the same time, the molecular design usually leads to the yellowish appearance and low transparency of self-healing materials.

Herein, the low-cost aliphatic bis (2-alkylethyl) disulfide (HEDS) with flexible segments was used to prepare the self-healing waterborne polyurethane materials, which was synthesized by introducing HEDS-containing - OH groups into the WPU and reacted with $-\mathrm{NCO}$ groups. The successful introduction of SS bond in the polymer chain was verified by FTIR. Then, the influence of SS bond content on the self-healing efficiency of WPU film was studied by the tensile test, the dispersion and stability of WPU were assessed by the particle size and zeta potential analysis and the thermal properties of the polymer were tested by DMA and TGA. In addition, the reprocessing performance of the film was investigated by a plate vulcanizing machine.

\section{Materials and Methods}

\subsection{Materials}

Poly-tetrahydrofuran (PTMG, Mn = 1000), Triethylamine (TEA), 2,2-Dimthylolpropionic acid (DMPA), Dibutyltin dilaurate (DBTDL) and bis (2-alkylethyl) disulfide (HEDS) were obtained from Shanghai Aladdin Company, Shanghai, China. Hexamethylene diisocyanate (HDI) was purchased from Bayer Technology, Germany. PTMG and DMPA should be dehumidified under vacuum at $100^{\circ} \mathrm{C}$ for $2 \mathrm{~h}$ before the experiment.

\subsection{Preparation of WPU-SS Emulsion}

WPU-SS was synthesized by three steps, in which acetone was replaced by DMAc to reduce the viscosity of polyurethane prepolymer. Table 1 lists the experimental formulation of different WPU samples. The molar ratio of the isocyanate group to hydroxyl group was kept at 1.4, and the ratio of PTMG to HEDS was 1/2 (WPU1), 1/1(WPU2) and 2/1(WPU3).

Table 1. Molar ratio formulations of different WPU samples.

\begin{tabular}{lccccc}
\hline & HDI & PTMG & DMPA & HEDS & TEA \\
\hline WPU1 & 3.5 & 0.55 & 0.85 & 1.10 & 0.85 \\
WPU2 & 3.5 & 0.83 & 0.85 & 0.83 & 0.85 \\
WPU3 & 3.5 & 1.10 & 0.85 & 0.55 & 0.85 \\
\hline
\end{tabular}

According to the experimental formulation listed in Table 1, the synthesis process of WPU2 was illustrated. As shown in Figure 1, firstly, PTMG and DMPA were added in a 500-mL four-necked round-bottomed flask with a mechanical agitator, nitrogen inlet and drying condenser tube. DMAc was used as a solvent, and DBTDL was used as a catalyst. IPDI was slowly added after thoroughly stirring. Under mechanical stirring, the reaction temperature was $80^{\circ} \mathrm{C}$, when the content of $-\mathrm{NCO}$ reached the theoretical value, the polyurethane prepolymer terminated with $-\mathrm{NCO}$ was generated. Then, the temperature was reduced to $60{ }^{\circ} \mathrm{C}$, HEDS containing $-\mathrm{OH}$ was added and reacted with isocyanate 
under nitrogen protection until the content of - $\mathrm{NCO}$ in the system reached the theoretical value again.<smiles>CCCCCCCC(=O)OCCOCCO</smiles>

PTMG

HDI<smiles>CC(CO)(CO)C(=O)O</smiles>

DMPA

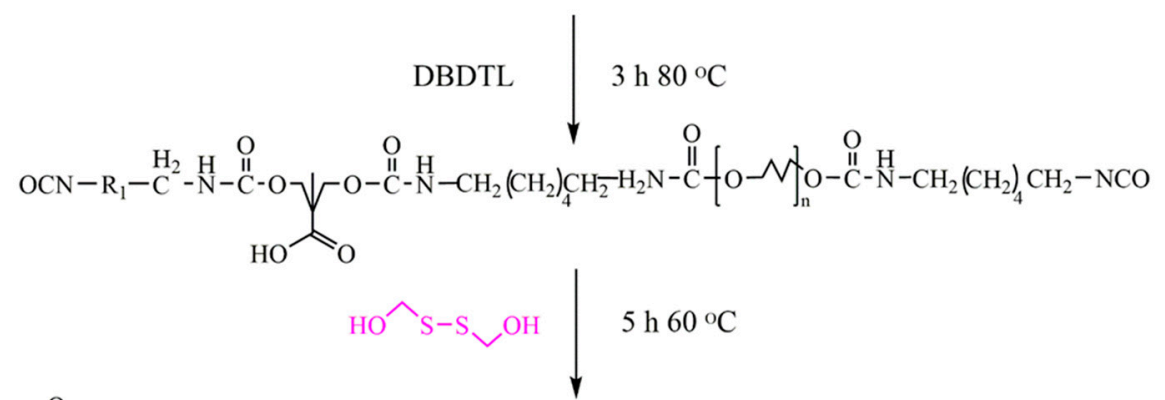

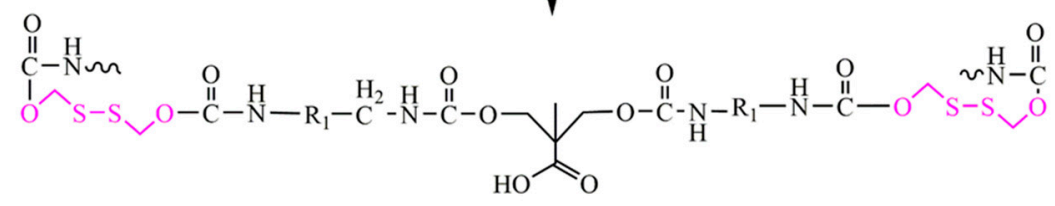

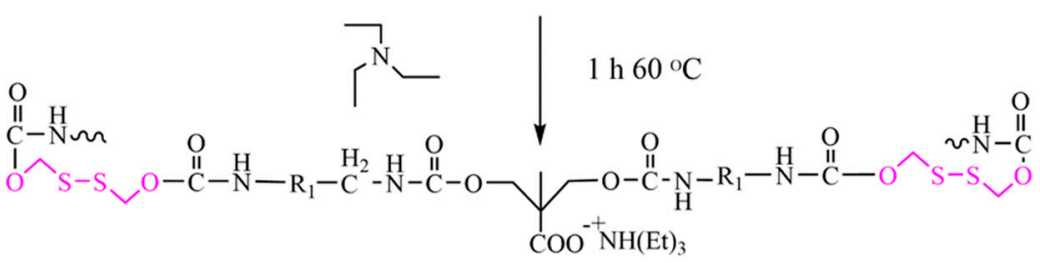

Figure 1. The synthesis process of WPU2 (R1 is the repeating chain segment).

TEA with DMPA and other substances was added to neutralize - $\mathrm{COOH}$ in DMPA, and the system temperature was further reduced to $6 \sim 10^{\circ} \mathrm{C}$. The synthesized mixture was added to deionized water for high-speed dispersion for $1 \mathrm{~h}$ to obtain the final WPU2 emulsion. In the emulsification process, the residual - NCO of the prepolymer reacted with deionized water to form urea and biuret derivatives. The final WPU2 emulsion was light blue and the solid content was about $30 \mathrm{wt} \%$. The WPU samples with PTMG and HEDS ratios of $1 / 2$ and $2 / 1$ were synthesized by the same method.

\subsection{Preparation of WPU Films}

The predetermined amount of WPU-SS emulsion was poured into the horizontally placed PTFE mold with the size of $10 \mathrm{~cm} \times 10 \mathrm{~cm} \times 1.5 \mathrm{~cm}$. After drying at room temperature for $48 \mathrm{~h}$, most of the water evaporated. Then, the film was stripped from the mold, and the water and solvent were completely removed within $24 \mathrm{~h}$ in a vacuum drying oven at $60^{\circ} \mathrm{C}$ to obtain a constant weight WPU-SS film. The prepared WPU-SS films were stored in the dryer containing silica gel before further characterization.

\subsection{Measurement of Isocyanate Content by Dibutyl-Amine Method}

(1) Reagent preparation:

a. Preparation of $0.1 \mathrm{~mol} / \mathrm{L}$ hydrochloric acid standard solution and calibration with anhydrous sodium carbonate.

b. Configure 0.1-mol/L di-n-butylamine-acetone solution: 12.9-g di-n-butylamine was placed in a 1000-mL volumetric flask, diluted with acetone and shaken. 
c. Bromocresol green indicator: 0.1-g bromocresol green was dissolved in 100-mL volumetric flasks with a $1.5-\mathrm{mL}$ concentration of $0.1-\mathrm{mol} / \mathrm{L}$ sodium hydroxide solution and diluted with distilled water to scale.

(2) Operation process: Accurate weighing 1 3-g prepolymer to 150-mL conical flask, adding 20-mL dibutyl-amine-acetone solution, fully reaction $20 \mathrm{~min}$ after adding 5 drops of bromocresol green indicator. Use the configured 0.1-mol/L hydrochloric acid standard solution to titrate the prepolymer. When the color changes from blue to yellow, it will be the end of the reaction. Read the volume and do a blank control experiment.

(3) Isocyanate content was determined by using the following Equation (1):

$$
-\mathrm{NCO} \%=\left[\left(V_{0}-V\right) \times \mathrm{c} \times 4.202\right] / \mathrm{m} \times 100 \%
$$

Thereinto, c is HCL concentration (mol/L), $V$ is the sample consumed HCL volume $(\mathrm{mL}), V_{0}$ is blank consumed HCL volume $(\mathrm{mL})$ and $\mathrm{m}$ is Sample mass $(\mathrm{g})$.

\subsection{Characterization}

\subsubsection{Determination of Dispersion Stability}

The average particle size and polydispersity index (PDI) of the dispersions were measured by dynamic light scattering (DLS) at room temperature using Zetasizer Nano ZS90 of Malvern Instrument and Equipment Company, Malvern, UK. In order to test the storage stability, all dispersions were stored in closed bottles and stored at room temperature.

\subsubsection{Fourier-Transform Infrared Spectroscopy (FTIR)}

The synthesized polymer was analyzed by TENSORII Fourier-transform infrared spectrometer produced by BRUKER company in Germany. The attenuated total reflection (ATR) mode was used for infrared testing of WPU-SS samples. The spectra obtained were recorded in the range of $4000-500 \mathrm{~cm}^{-1}$.

\subsubsection{Dynamic Thermomechanical Analysis (DMA)}

The dynamic mechanical properties of the rectangular WPU-SS spline $(30 \mathrm{~mm} \times 10 \mathrm{~mm})$ were tested using the tensile mode of the DMA 850 dynamic thermomechanical analyzer of the United States TA company. The test temperature range is $-100 \sim 120^{\circ} \mathrm{C}$, and the experimental frequency is $1 \mathrm{~Hz}$. The heating rate is $5^{\circ} \mathrm{C} / \mathrm{min}$.

\subsubsection{Thermogravimetric Analysis (TGA)}

A certain amount of WPU-SS was investigated by the TGA55 thermogravimetric analyzer of TA company. It was carried out in a nitrogen atmosphere with an airflow velocity of $20 \mathrm{~mL} / \mathrm{min}$, a heating rate of $10^{\circ} \mathrm{C} / \mathrm{min}$ and a temperature range of $30 \sim 800{ }^{\circ} \mathrm{C}$.

\subsubsection{X-ray Photoelectron Spectroscopy (XPS)}

The surface chemical compositions of WPU-SS samples were analyzed by XPS ESCALAB 250A of Thermo Electron Corporation company with an Al K $\alpha$ excitation radiation. The containment $\mathrm{C}$ 1s hydrocarbon peak at $284.8 \mathrm{eV}$ was applied to calibrate the binding energies.

\subsubsection{Determination of Self-Healing Performance}

The samples were cut into dumbbell-shaped samples, and five points were randomly selected on the dumbbell-shaped samples to measure the thickness using a desktop thickness gauge, and the average value was taken. The USA Instron 3369 universal tensile testing machine was used to test the tensile strength and elongation at break at $100 \mathrm{~mm} / \mathrm{min}$, and the average value of each sample was measured after 5 times.

The dumbbell-shaped standard sample was cut from the middle position to part of the adhesion, and then the incision part was docked together. The samples were repaired 
under different conditions. The tensile properties of original and repaired samples were tested, and the self-healing efficiency was calculated by using the following Equation (2):

$$
\eta \%=\sigma / \sigma_{0} \times 100 \%
$$

where $\eta$ is self-healing efficiency, $\sigma$ is fracture strength of repaired specimen and $\sigma_{0}$ is fracture strength of the original sample.

\section{Results and Discussion}

\subsection{Dispersion and Stability Analysis}

The synthesized WPU-SS emulsion was analyzed by a nano-particle size analyzer to evaluate its storage stability. The particle size and distribution of suspension waterborne polyurethane particles are shown in Figure 2a. The average particle sizes of WPU1, WPU2 and WPU3 were 71.27, 77.12 and $131.6 \mathrm{~nm}$, respectively, and the PDI were $0.130,0.084$ and 0.285 , respectively. The particle sizes of WPU1 and WPU2 dispersion systems are similar, and the polydispersity index shows that the distribution of WPU2 dispersion is more uniform than that of WPU1 dispersion. With the increase of soft segment content, the proportion of hydrophilic groups in WPU3 emulsion decreased, the polydispersity index increased, and the dispersion became worse.
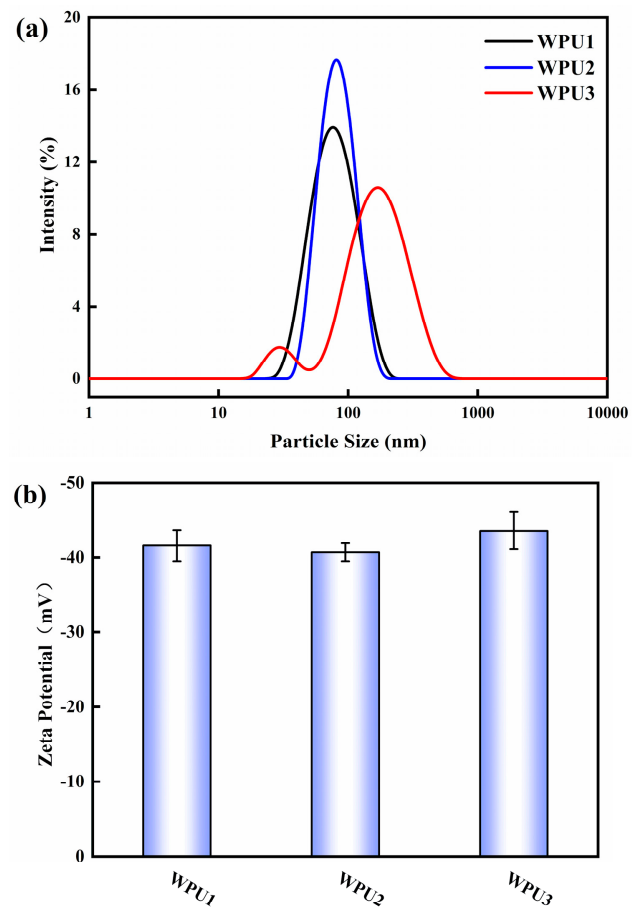

Figure 2. Particle size and distribution of WPU (a) and their zeta potential (b).

Zeta potential is another important indicator for evaluating emulsion stability. As shown in Figure 2b, the Zeta potential of WPU1, WPU2 and WPU3 were $-41.6,-40.7$ and $-43.6 \mathrm{mV}$, respectively. The high absolute value of zeta potential indicates that there is abundant electrostatic repulsion on the surface of water particles. Besides, introducing SS bonds in the WPU-SS chain had little effect on the Zeta potential value, manifesting that the addition of SS bonds had no significant effect on the stability of the emulsion. In addition, there is no deposit at the bottom of WPU-SS dispersions stored in closed glass bottles at room temperature for 2 months. The WPU emulsions were diluted into $1 \mathrm{wt} \%$ water dispersion and looked light blue. In summary, stable WPU emulsions can be obtained by introducing SS bond into the molecule chain. 


\subsection{Structure Analysis}

Figure 3 are FTIR spectra and XPS survey spectra of the linear WPU-SS samples. In Figure 3a, all curves have no absorption peak at $2270 \mathrm{~cm}^{-1}$, which indicates that $-\mathrm{NCO}$ groups in the system are all involved in the reaction $[16,17]$. Compared with the three curves, the distribution of all characteristic peaks was essentially the same, indicating that $-\mathrm{OH}$ and $-\mathrm{NCO}$ on HEDS reacted to form carbamate, and no new chemical bond was formed. The three curves are in line with the characteristic peaks of waterborne polyurethane. The band located at $3321 \mathrm{~cm}^{-1}$ corresponds to the $\mathrm{N}-\mathrm{H}$ stretching vibration and the band at $1531 \mathrm{~cm}^{-1}$ is assigned to the $\mathrm{N}-\mathrm{H}$ in-plane bending vibration $[18,19]$. The absorption bands at 1460 and $1360 \mathrm{~cm}^{-1}$ are assigned to the $-\mathrm{CH}_{2}$ - bending vibrations. The bands at 2940 and $2856 \mathrm{~cm}^{-1}$ are associated with the $\mathrm{C}-\mathrm{H}$ asymmetry and symmetric stretching vibration of methylene in polyurethane molecular chain $[20,21]$. The band at $1110 \mathrm{~cm}^{-1}$ corresponds to bending vibration of $\mathrm{C}-\mathrm{O}$ (aliphatic ether) in polyether polyol [22]. The characteristic band about 1707 and $1631 \mathrm{~cm}^{-1}$ is related with nonhydrogen bond $\mathrm{C}=\mathrm{O}$ hydrogen bond $\mathrm{C}=\mathrm{O}$ in urea, respectively [23-25]. HEDS in polymer chain has a characteristic absorption peak of SS bond at 637 [26]. With the increase of disulfide bond content, the absorption peak of the corresponding SS bond at $637 \mathrm{~cm}^{-1}$ became more obvious, which proved that SS bond was successfully introduced into waterborne polyurethane to form WPU-SS.
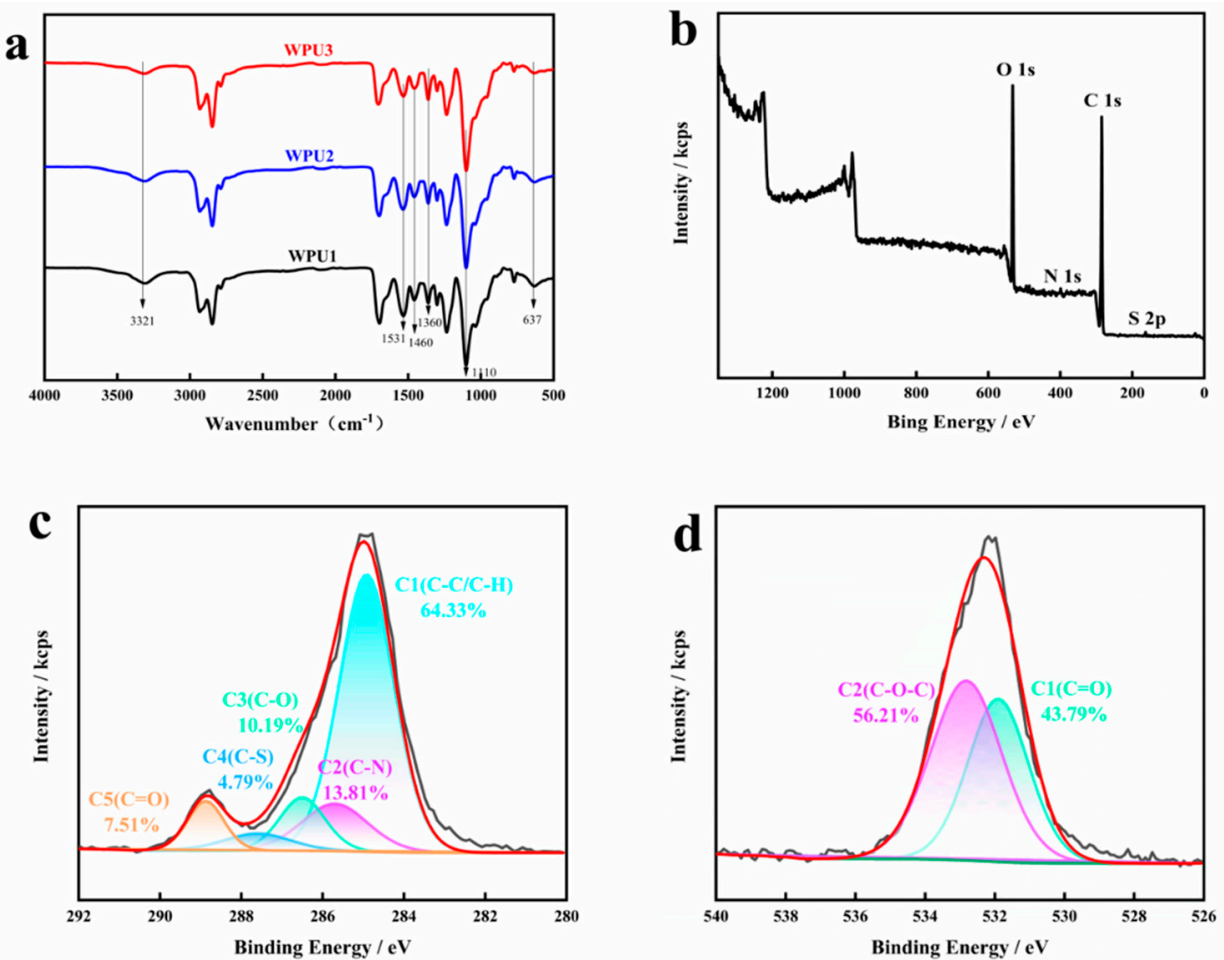

Figure 3. (a) FTIR spectra of WPU-SS samples, (b) XPS survey spectra of WPU2, (c) C 1s of WPU2, and (d) O 1s of WPU2.

The XPS measurement was used to further confirm the construction by analyzing the chemical compositions in the WPU-SS system. The peaks in XPS survey spectrum indicated the present of $S$ element $(163.5 \mathrm{eV})$ in the samples due to disulfide bond should be successfully chemically linked to the matrix of waterborne polyurethane. In addition, the $C$ 1s spectrum was further measured to explore the types of carbon bonds to analyze the existence of interfacial interactions, as shown in Figure 3c. The $\mathrm{C} 1 \mathrm{~s}$ peak was curvedfitted into four main components including $\mathrm{C}-\mathrm{C} / \mathrm{C}-\mathrm{H}, \mathrm{C}-\mathrm{N}, \mathrm{C}-\mathrm{O}, \mathrm{C}-\mathrm{S}$ and $\mathrm{C}=\mathrm{O}$ bonds corresponding to the peaks at $284.8,285.7,286.5,287.7$ and $288.8 \mathrm{eV}$, respectively. The $\mathrm{O}$ 1 s peak was curved-fitted into four main components including $\mathrm{C}-\mathrm{O}-\mathrm{C}$ and $\mathrm{C}=\mathrm{O}$ bonds. 
The peak of C-S bond appeared implying that disulfide bond was successfully grafted onto polyurethane chains, as supported by FTIR results.

\subsection{Dynamic Thermodynamic Analysis}

The DMA results are shown in Figure 4. During the heating process of WPU-SS at $-100 \sim 120^{\circ} \mathrm{C}$, the polymer with microphase separation structure showed two glass transition temperatures. The storage modulus and loss modulus of WPU-SS films are shown in Figure $4 a, b$, respectively. It could be found that the storage modulus $\left(E^{\prime}\right)$ decreased significantly at about -70 to $-60^{\circ} \mathrm{C}$, while the loss modulus ( $\left.\mathrm{E}^{\prime \prime}\right)$ increased significantly at the same temperature range, which corresponding to the glass transition of the hard segments, moreover, with the increase of SS bonds, the $\mathrm{E}^{\prime}$ did not change much while the peak value of E" changed obviously, which meant the move of the polymer chains took more energy. The decrease of disulfide bond content leads to the increase of soft segment content, which reduced the $E^{\prime}$ and $E^{\prime \prime}$ of the films at room temperature. This is mainly due to the less restriction of the slip of the soft segment molecular chain, which leads to the easier entanglement of the molecular chain and the easier interaction with the main chain [27]. At the same time, the degree of microphase separation is reduced but the damping performance is improved.
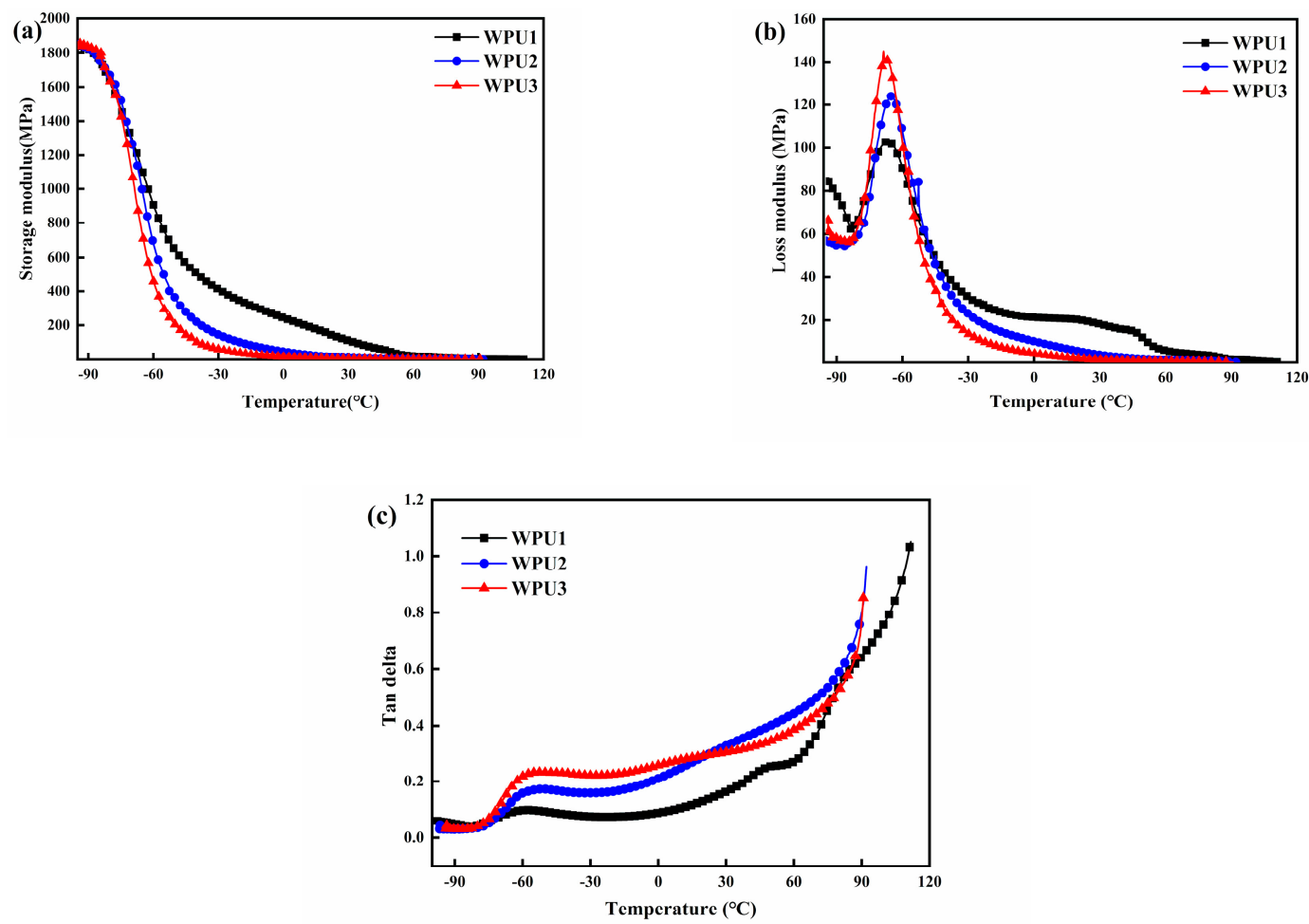

Figure 4. (a) Storage modulus, (b) loss modulus and (c) tan delta of WPU-SS samples with different contents of SS as a function of temperature.

The link between tan delta and temperature is determined in Figure 4c. With the loss of SS bond loading, the soft segment rises, and the microphase separation diminishes gradually. When the microphase separation is cut down to a certain extent, the glass transition temperature of the hard segment cannot be shown in the experiment. Since the glass transition temperature of the hard segment is much lower than room temperature, the film is in a highly elastic state and exhibits the properties of an elastomer at room temperature. 


\subsection{Thermal Stability Analysis}

To investigate the thermal stability of WPU-SS films, thermogravimetric analysis (TGA) was carried out at $30 \sim 800{ }^{\circ} \mathrm{C}$ in a nitrogen atmosphere. Figure 5 shows the typical TGA and the first derivative curve DTG, respectively. The characteristic thermal degradation data display in Table 2. From the TGA curve, the thermal stability of WPU-SS films was improved with the decrease of short chain disulfide bond content and the enhancement of long chain PTMG content. In particular, the $50 \%$ weight loss temperature $\left(\mathrm{T}_{\mathrm{d}} 50 \%\right)$ increased from $361{ }^{\circ} \mathrm{C}$ to $382{ }^{\circ} \mathrm{C}$. In the region of $230 \sim 440{ }^{\circ} \mathrm{C}$, there are two obvious degradation stages for WPU-SS films. The partial weight loss at $230 \sim 350{ }^{\circ} \mathrm{C}$ can be attributed to the rupture of the amino ester bond in the hard segment isocyanate, and the thermal stability of the hard segment is poor, which can be decomposed into primary amines (or secondary amines), alkenes and carbon dioxide [28-30]. In contrast, polyether has better thermal stability, $350 \sim 440{ }^{\circ} \mathrm{C}$ weight loss is mainly the decomposition of the soft segment [31-33].

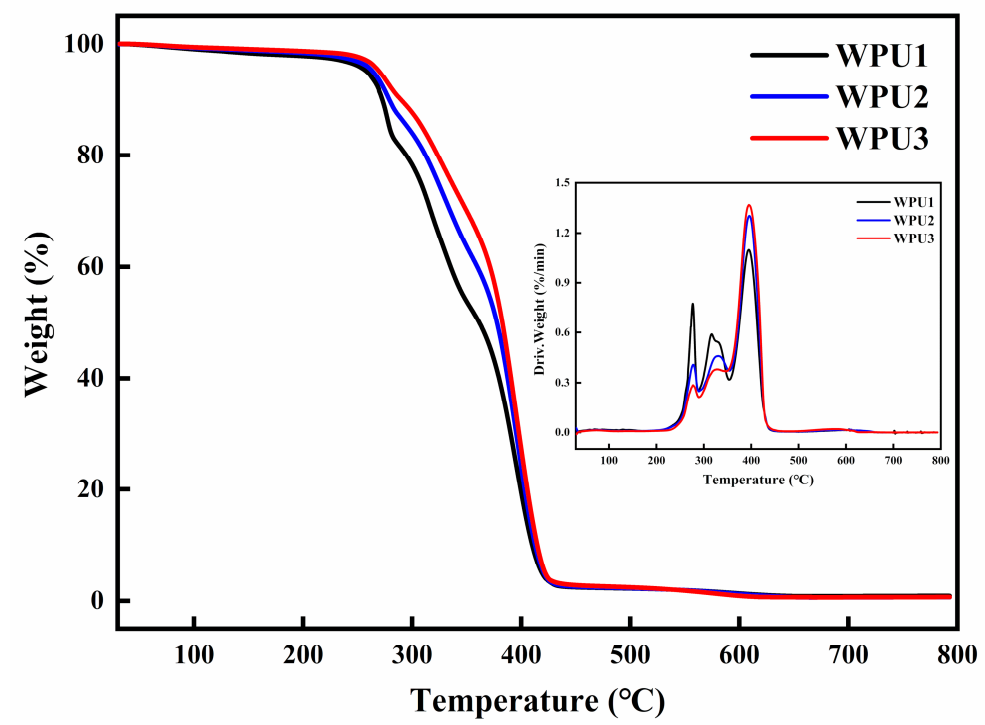

Figure 5. TGA curves and DTG curves of different WPU-SS films.

Table 2. Particular thermal degradation dates of diifferent WPU samples.

\begin{tabular}{cccc}
\hline & $\mathrm{T}_{\mathbf{d}} \mathbf{5 \%}\left({ }^{\circ} \mathbf{C}\right)$ & $\mathbf{T}_{\mathbf{d}} \mathbf{1 0} \%\left({ }^{\circ} \mathbf{C}\right)$ & $\mathbf{T}_{\mathbf{d}} \mathbf{5 0} \%\left({ }^{\circ} \mathbf{C}\right)$ \\
\hline WPU1 & 256 & 273 & 361 \\
WPU2 & 264 & 278 & 377 \\
WPU3 & 270 & 290 & 382 \\
\hline
\end{tabular}

\subsection{Tensile Properties and Self-Healing Efficiency of WPU-SS Films}

In addition, the self-healing process of WPU films was evaluated more quantitatively through tensile measurement, and the self-healing efficiency was calculated according to the final tensile stress ratio of the repaired sample to the original sample. Figure 6 reveals the stress-strain curves of the original samples of WPU1, WPU2 and WPU3. The film of WPU1 exhibits a high tensile strength since the hard segment content is relatively high, and the content of carbamate in WPU1 may be more than that in WPU2 and WPU3. On the contrary, WPU2 and WPU3 possesses high soft segment content, and strong molecular weight mobility, so its self-healing performance is also relatively improved. 


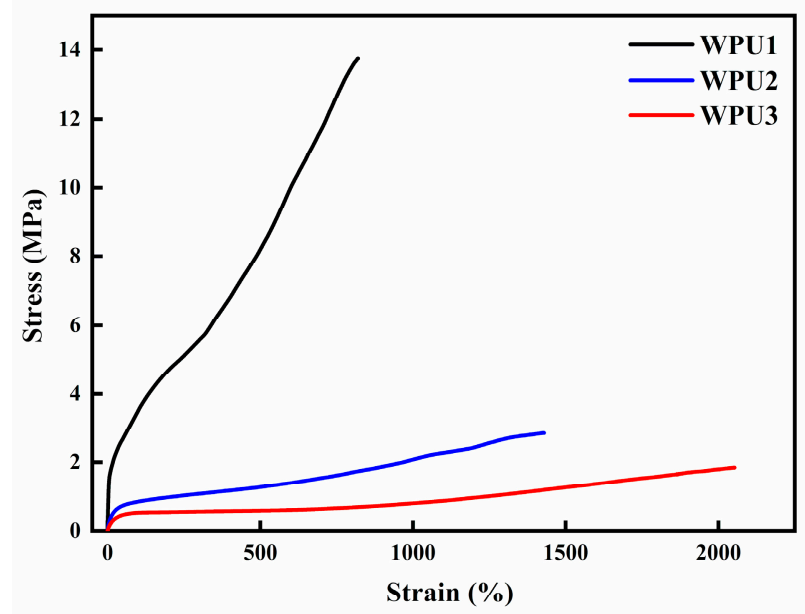

Figure 6. Stress-strain curve of WPU1, WPU2, WPU3.

Figure 7 shows the self-healing efficiency of WPU1 WPU2 and WPU3 under different conditions. The self-healing efficiency of WPU1 is only $29.02 \%$ after $4 \mathrm{~h}$ at $70{ }^{\circ} \mathrm{C}$, while the healing efficiency of the WPU2 and WPU3 films reached $96.14 \%$ and $97.28 \%$ under the same conditions, respectively. Meanwhile, WPU2 and WPU3 films also had a self-healing efficiency of up to $84.21 \%$ and $85.86 \%$ after being placed at $25^{\circ} \mathrm{C}$ for $24 \mathrm{~h}$. The table of repair efficiency changing over time is shown in Table 3. There is no doubt that introducing SS bond endowed the film with excellent self-healing performance. Of course, the content of the soft segment also exerts a certain degree of influence on the repair effect of the film $[34,35]$.
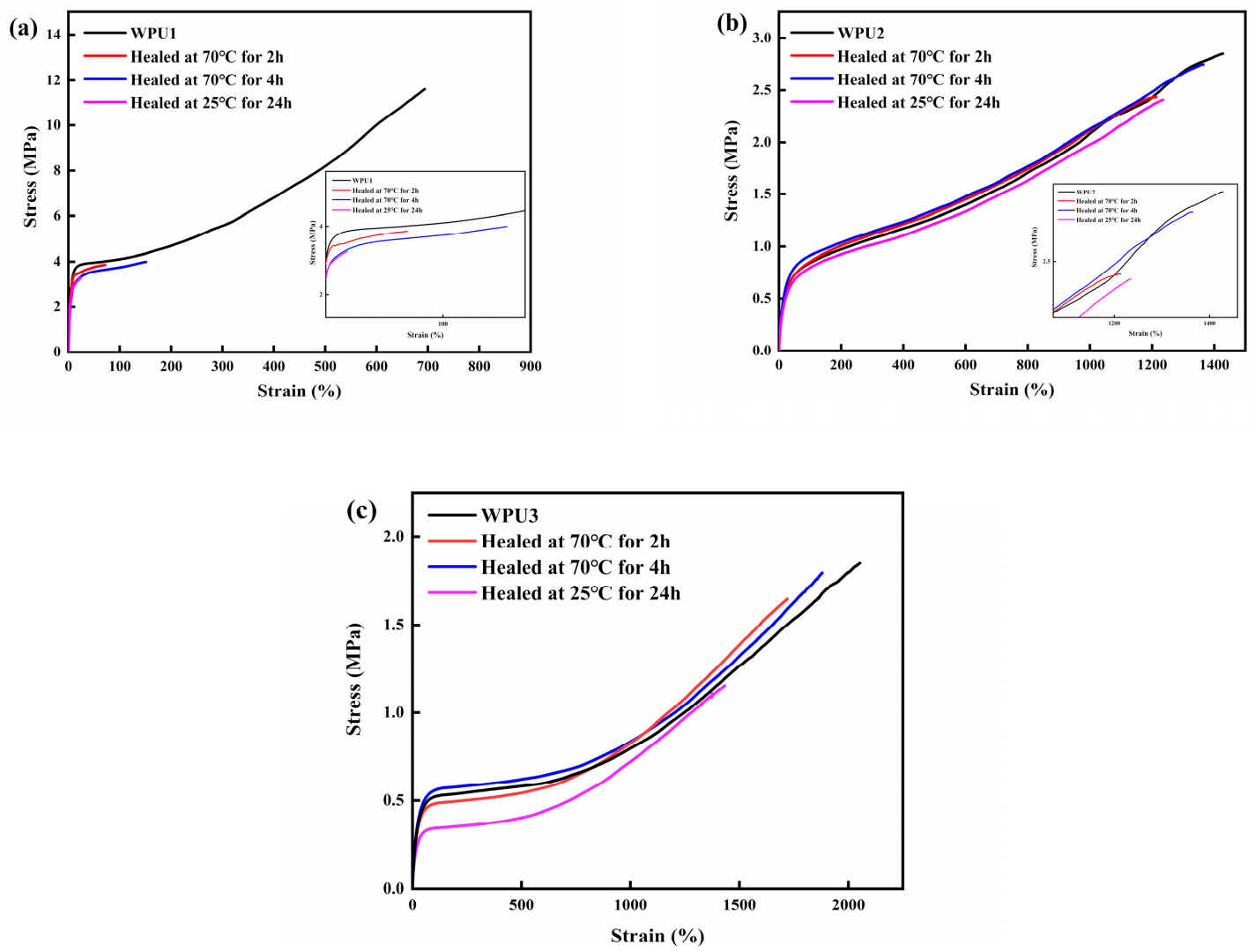

Figure 7. Stress-strain curves of WPU1 (a), WPU2 (b) and WPU3 (c) films repaired under different conditions. 
Table 3. Details of Tensile Results and the Healing Efficiency of WPU-SS Samples under different conditions.

\begin{tabular}{ccccc}
\hline Samples & $\begin{array}{c}\text { Heat Treatment } \\
\text { Time (h) }\end{array}$ & $\begin{array}{c}\text { Tensile } \\
\text { Strength (MPa) }\end{array}$ & $\begin{array}{c}\text { Elongation at } \\
\text { Break (\%) }\end{array}$ & $\begin{array}{c}\text { Healing } \\
\text { Efficiency (\%) }\end{array}$ \\
\hline WPU1 & $/$ & 13.75 & 820.01 & $/$ \\
& 2 & 3.85 & 71.67 & 28.00 \\
& 4 & 3.99 & 151.12 & 29.02 \\
WPU2 & $24\left(25^{\circ} \mathrm{C}\right)$ & 3.26 & 23.9 & 23.70 \\
& $/$ & 2.85 & 1427.78 & $/$ \\
& 2 & 2.43 & 1213.34 & 85.26 \\
WPU3 & 4 & 2.74 & 1365.01 & 96.14 \\
& $24\left(25^{\circ} \mathrm{C}\right)$ & 1.40 & 1235.01 & 84.21 \\
& $/$ & 1.64 & 2053.08 & $/$ \\
& 2 & 1.79 & 1719.64 & 89.13 \\
& 4 & 1.58 & 1880.01 & 97.28 \\
& $24\left(25^{\circ} \mathrm{C}\right)$ & & 1671.88 & 85.86 \\
\hline
\end{tabular}

\subsection{Reprocessing Performance}

After being placed in a constant temperature and humidity box for $24 \mathrm{~h}$, the cut dumbbell-shaped spline was used for the tensile test. Figure 8 provides the stress-strain curve of WPU2 film and WPU2 film after reprocessing. The tensile strength of WPU2 film after reprocessing was $3.39 \mathrm{MPa}$, compared with the original spline, its tensile strength increased and the elongation at break reduced, which indicated that the film had a good reprocessing performance.

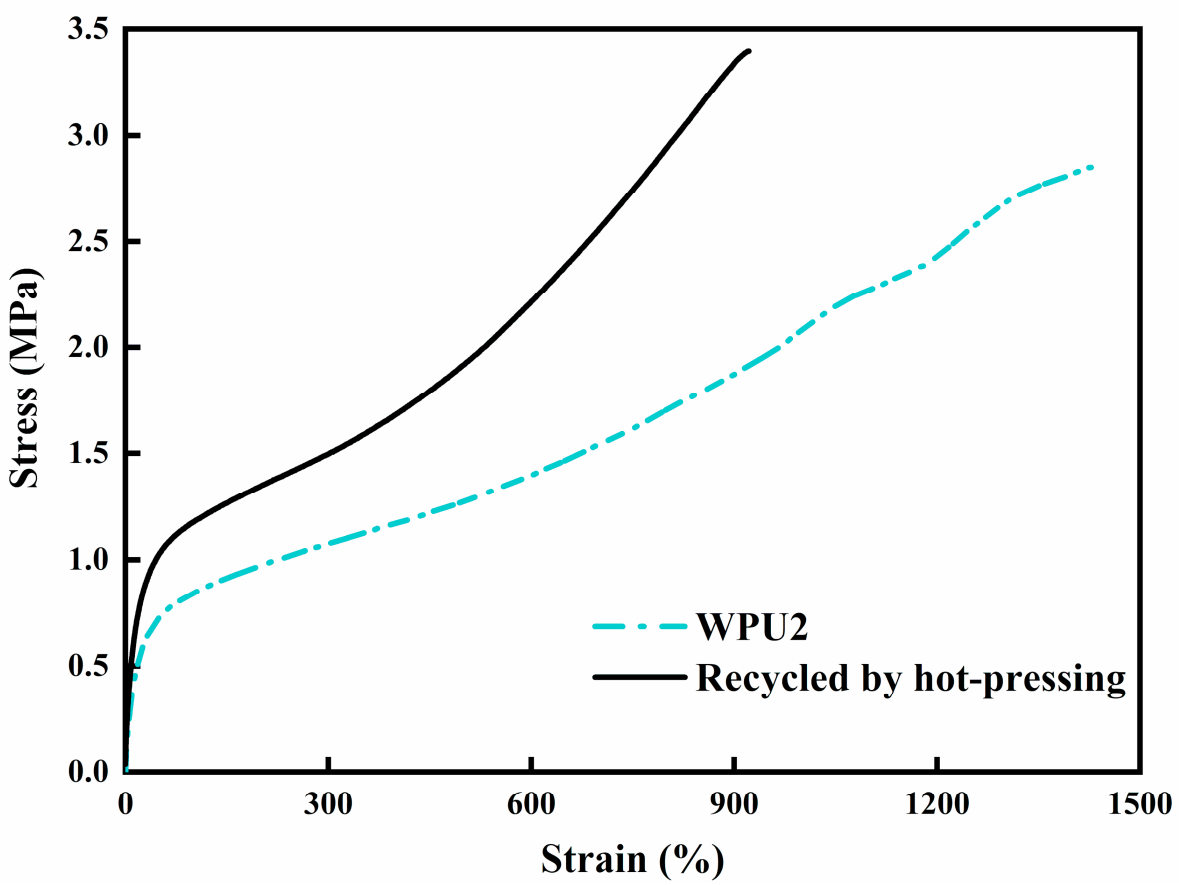

Figure 8. Tensile properties of the reprocessed WPU2 film.

\section{Conclusions}

In this paper, based on the principle of introducing reversible covalent bond containing SS into polymer, functional self-healing waterborne polyurethane was synthesized with PTMG as soft segment, IPDI as hard segment and DMPA containing hydrophilic group as auxiliary. The zeta potential revealed that all the synthesized WPU emulsions displayed excellent stability, and the particle sizes of WPU1 and WPU2 emulsions were relatively small. The characteristic peaks of N-H and S-S in urethane were verified by FTIR. Furthermore, the tensile strength, self-healing process and repair effect of WPU-SS film 
were quantitatively evaluated by tensile measurement. After the cut spline WPU2 sample was heat treated at $70{ }^{\circ} \mathrm{C}$ for $4 \mathrm{~h}$, it could maintain the tensile strength and the self-healing efficiency could reach $96.14 \%$, whilst the repair efficiency of the sample was $84.21 \%$ after 24-h self-repair at $25^{\circ} \mathrm{C}$. In addition, to realize the reprocessing of the self-healing film, the cut WPU2 film fragments were hot pressed at $130{ }^{\circ} \mathrm{C}$ for $20 \mathrm{~min}$ to reshape into a square film by a flat vulcanizing machine. The results indicated that the tensile strength of the reprocessed WPU2 film was $3.39 \mathrm{MPa}$, and it possessed good reprocessing performance.

Author Contributions: G.Y. conceived the project and wrote the paper; T.J. was in charge of all the experiments, all the authors contributed to the scientific discussion. Both authors have read and agreed to the published version of the manuscript.

Funding: This research received no external funding.

Institutional Review Board Statement: Not applicable.

Informed Consent Statement: Not applicable.

Data Availability Statement: Not applicable.

Acknowledgments: The authors also would like to thank Yunyun Zou from the Shiyanjia lab for the XPS analysis (www.Shiyanjia.com, accessed on 19 August 2021).

Conflicts of Interest: The authors declare no conflict of interest.

\section{References}

1. Ur Rehman, H.; Chen, Y.; Hedenqvist, M.S.; Li, H.; Xue, W.; Guo, Y.; Guo, Y.; Duan, H.; Liu, H. Self-Healing Shape Memory PUPCL Copolymer with High Cycle Life. Adv. Funct. Mater. 2018, 28, 1704109. [CrossRef]

2. Zhou, L.; Liu, Q.; Lv, X.; Gao, L.; Fang, S.; Yu, H. Photoinduced triple shape memory polyurethane enabled by doping with azobenzene and GO. J. Mater. Chem. C 2016, 4, 9993-9997. [CrossRef]

3. Heo, Y.; Sodano, H.A. Self-Healing Polyurethanes with Shape Recovery. Adv. Funct. Mater. 2014, 24, 5261-5268. [CrossRef]

4. Kim, J.; Hong, P.H.; Choi, K.; Moon, G.; Kang, J.; Lee, S.; Lee, S.; Jung, H.W.; Ko, M.J.; Hong, S.W. A Heterocyclic Polyurethane with Enhanced Self-Healing Efficiency and Outstanding Recovery of Mechanical Properties. Polymers 2020, 12, 968. [CrossRef] [PubMed]

5. Yang, Y.; Lu, X.; Wang, W. A tough polyurethane elastomer with self-healing ability. Mater. Des. 2017, 127, 30-36. [CrossRef]

6. Hillewaere, X.K.D.; Du Prez, F.E. Fifteen chemistries for autonomous external self-healing polymers and composites. Prog. Polym. Sci. 2015, 49-50, 121-153. [CrossRef]

7. Campanella, A.; Dohler, D.; Binder, W.H. Self-Healing in Supramolecular Polymers. Macromol. Rapid Commun. 2018, 39, 1700739. [CrossRef] [PubMed]

8. $\quad$ Fan, L.F.; Rong, M.Z.; Zhang, M.Q.; Chen, X.D. Dynamic reversible bonds enable external stress-free two-way shape memory effect of a polymer network and the interrelated intrinsic self-healability of wider crack and recyclability. J. Mater. Chem. A 2018, 6, 16053-16063. [CrossRef]

9. Zhou, L.; Chen, M.; Zhao, X. Rapid degradation of disulfide-based thermosets through thiol-disulfide exchange reaction. Polymers 2017, 120, 1-8. [CrossRef]

10. Cordier, P.; Tournilhac, F.; Soulié-Ziakovic, C.; Leibler, L. Self-healing and thermoreversible rubber from supramolecular assembly. Nature 2008, 451, 977-980. [CrossRef]

11. de Luzuriaga, A.R.; Martin, R.; Markaide, N.; Rekondo, A.; Cabañero, G.; Rodríguez, J.; Odriozola, I. Correction: Epoxy resin with exchangeable disulfide crosslinks to obtain reprocessable, repairable and recyclable fiber-reinforced thermoset composites. Mater. Horiz. 2020, 7, 2460-2461. [CrossRef]

12. Sarma, R.J.; Otto, S.; Nitschke, J.R. Disulfides, imines, and metal coordination within a single system: Interplay between three dynamic equilibria. Chemistry 2007, 13, 9542-9546. [CrossRef] [PubMed]

13. Rekondo, A.; Martin, R.; de Luzuriaga, A.R.; Cabañero, G.; Grande, H.J.; Odriozola, I. Catalyst-free room-temperature self-healing elastomers based on aromatic disulfide metathesis. Mater. Horiz. 2014, 1, 237-240. [CrossRef]

14. Kim, S.M.; Jeon, H.; Shin, S.H.; Park, S.A.; Jegal, J.; Hwang, S.Y.; Oh, D.X.; Park, J. Superior Toughness and Fast Self-Healing at Room Temperature Engineered by Transparent Elastomers. Adv. Mater. 2018, 30, 1705145. [CrossRef]

15. Xu, W.M.; Rong, M.Z.; Zhang, M.Q. Sunlight driven self-healing, reshaping and recycling of a robust, transparent and yellowingresistant polymer. J. Mater. Chem. A 2016, 4, 10683-10690. [CrossRef]

16. Zhang, P.; Lu, Y.; Fan, M.; Jiang, P.; Dong, Y. Modified cellulose nanocrystals enhancement to mechanical properties and water resistance of vegetable oil-based waterborne polyurethane. J. Appl. Polym. Sci. 2019, 136, 48228. [CrossRef]

17. Kim, M.S.; Ryu, K.M.; Lee, S.H.; Choi, Y.C.; Rho, S.; Jeong, Y.G. Chitin Nanofiber-Reinforced Waterborne Polyurethane Nanocomposite Films with Enhanced Thermal and Mechanical Performance. Carbohydr. Polym. 2021, 258, 117728. [CrossRef] [PubMed] 
18. Hadjadj, A.; Jbara, O.; Tara, A.; Gilliot, M.; Malek, F.; Maafi, E.M.; Tighzert, L. Effects of cellulose fiber content on physical properties of polyurethane based composites. Compos. Struct. 2016, 135, 217-223. [CrossRef]

19. Lin, C.K.; Kuo, J.F.; Chen, C.Y.; Fang, J.J. Investigation of bifurcated hydrogen bonds within the thermotropic liquid crystalline polyurethanes. Polymers 2012, 53, 254-258. [CrossRef]

20. Wu, W.; Zhao, W.; Gong, X.; Sun, Q.; Cao, X.; Su, Y.; Yu, B.; Li, R.K.; Vellaisamy, R.A. Surface decoration of halloysite nanotubes with POSS for fire-safe thermoplastic polyurethane nanocomposites. J. Mater. Sci. Technol. 2021. [CrossRef]

21. Zhao, H.; Zhao, S.Q.; Hu, G.H.; Zhang, Q.C.; Liu, Y.; Huang, C.X.; Li, W.; Jiang, T.; Wang, S.F. Synthesis and characterization of waterborne polyurethane/polyhedral oligomeric silsesquioxane composites with low dielectric constants. Polym. Adv. Technol. 2019, 30, 2313-2320. [CrossRef]

22. Lu, W.C.; Chuang, F.S.; Venkatesan, M.; Cho, C.J.; Chen, P.Y.; Tzeng, Y.R.; Yu, Y.Y.; Rwei, S.P.; Kuo, C.C. Synthesis of Water Resistance and Moisture-Permeable Nanofiber Using Sodium Alginate-Functionalized Waterborne Polyurethane. Polymers 2020, 12, 2882. [CrossRef] [PubMed]

23. Gubanska, I.; Kucinska-Lipka, J.; Janik, H. The influence of amorphous macrodiol, diisocyanate type and l-ascorbic acid modifier on chemical structure, morphology and degradation behavior of polyurethanes for tissue scaffolds fabrication. Polym. Degrad. Stab. 2019, 163, 52-67. [CrossRef]

24. Gao, Z.; Peng, J.; Zhong, T.; Sun, J.; Wang, X.; Yue, C. Biocompatible elastomer of waterborne polyurethane based on castor oil and polyethylene glycol with cellulose nanocrystals. Carbohydr. Polym. 2012, 87, 2068-2075. [CrossRef]

25. Zhao, H.; Zhao, S.Q.; Li, Q.; Khan, M.R.; Liu, Y.; Lu, P.; Huang, C.X.; Huang, L.J.; Jiang, T. Fabrication and properties of waterborne thermoplastic polyurethane nanocomposite enhanced by the POSS with low dielectric constants. Polymers 2020, $209,122992$. [CrossRef]

26. Zhao, D.; Liu, S.; Wu, Y.; Guan, T.; Sun, N.; Ren, B. Self-healing UV light-curable resins containing disulfide group: Synthesis and application in UV coatings. Prog. Org. Coat. 2019, 133, 289-298. [CrossRef]

27. Zhao, H.; Hao, T.H.; Hu, G.H.; Shi, D.; Huang, D.; Jiang, T.; Zhang, Q.C. Preparation and Characterization of Polyurethanes with Cross-Linked Siloxane in the Side Chain by Sol-Gel Reactions. Materials 2017, 10, 247. [CrossRef] [PubMed]

28. Zhao, H.; Huang, D.; Hao, T.H.; Hu, G.H.; Ye, G.B.; Jiang, T.; Zhang, Q.C. Synthesis and Investigation of Well-Defined Silane Terminated and Segmented Waterborne Hybrid Polyurethanes. New J. Chem. 2017, 41, 9268-9275. [CrossRef]

29. Chattopadhyay, D.K.; Webster, D.C. Thermal stability and flame retardancy of polyurethanes. Prog. Polym. Sci. 2009, 34, 1068-1133. [CrossRef]

30. Floros, M.; Hojabri, L.; Abraham, E.; Jose, J.; Thomas, S.; Pothan, L.; Leao, A.L.; Narine, S. Enhancement of thermal stability, strength and extensibility of lipid-based polyurethanes with cellulose-based nanofibers. Polym. Degrad. Stab. 2012, 97, 1970-1978. [CrossRef]

31. Fu, H.; Wang, Y.; Li, X.; Chen, W. Synthesis of vegetable oil-based waterborne polyurethane/silver-halloysite antibacterial nanocomposites. Compos. Sci. Technol. 2016, 126, 86-93. [CrossRef]

32. Liu, N.; Zhao, Y.; Kang, M.; Wang, J.; Wang, X.; Feng, Y.; Yin, N.; Li, Q. The effects of the molecular weight and structure of polycarbonatediols on the properties of waterborne polyurethanes. Prog. Org. Coat. 2015, 82, 46-56. [CrossRef]

33. Trovati, G.; Sanches, E.A.; Neto, S.C.; Mascarenhas, Y.P.; Chierice, G.O. Characterization of polyurethane resins by FTIR, TGA, and XRD. J. Appl. Polym. Sci. 2010, 115, 263-268. [CrossRef]

34. Zhao, H.; She, W.; Shi, D.; Wu, W.; Zhang, Q.C.; Li, R.K. Polyurethane/POSS nanocomposites for superior hydrophobicity and high ductility. Compos. Part B Eng. 2019, 177, 107441. [CrossRef]

35. Lai, Y.; Kuang, X.; Zhu, P.; Huang, M.; Dong, X.; Wang, D. Colorless, Transparent, Robust, and Fast Scratch-Self-Healing Elastomers via a Phase-Locked Dynamic Bonds Design. Adv. Mater. 2018, 30, 1802556. [CrossRef] [PubMed] 